\title{
Analytical investigation of ice floe drift in the marginal ice zone
}

\author{
R. M. Abid ${ }^{1}$, S. H. Mousavizadegan ${ }^{2}$ \& M. Rahman ${ }^{3}$ \\ ${ }^{1}$ Department of Information Technology, Schneider National, Inc., USA \\ ${ }^{2}$ Faculty of Marine Technology, Amirkabir University of Technology, Iran \\ ${ }^{3}$ Department of Engineering Mathematics, Dalhousie University, Canada
}

\begin{abstract}
Analytical solutions were constructed to investigate the ice floe drift, velocity field, and trajectories. The mathematical model considers the balance of atmosphere and ocean drag forces on ice floe, including skin and body drag forces from wind, waves, and currents. We have obtained numerical solutions to our mathematical models of air-ice stress. Graphical solutions are presented for ice floe drift due to wind stress. Mathematical formulations are being developed for the ice floe drift due to Eulerian current, water-ice form stress, and wave radiation pressure. We systematically presented in this paper the classical solutions of the ice floe drift, velocity, and trajectories considering the effects of wind. Numerical solutions of the mathematical models developed here, have been computed and presented. The mathematical models will be tested with available experimental data.

Keywords: ice floe, marginal ice zone, MIZ, ice drift, ocean surface waves, energy balance equation, wave spectrum, ice floe velocity components, ice floe trajectories.
\end{abstract}

\section{Introduction}

An ice floe is a floating chunk of sea ice that is less than 10 kilometers in its greatest dimension. Marginal Ice Zone (MIZ) is an interfacial region of ice floes which forms at the boundary of open water and the continuous ice pack. Figure 1 depicts a typical MIZ situation including ice floes and wave induced ice fracture at the ice edge. This paper considers the mathematical model for the ice edge and ice floe trajectories which is based on a balance equation for forces due to wind, waves, and currents impinging on the ice, as described by Tang and Fissel [7], Steele et al. [6] and Jenkins [3]. 

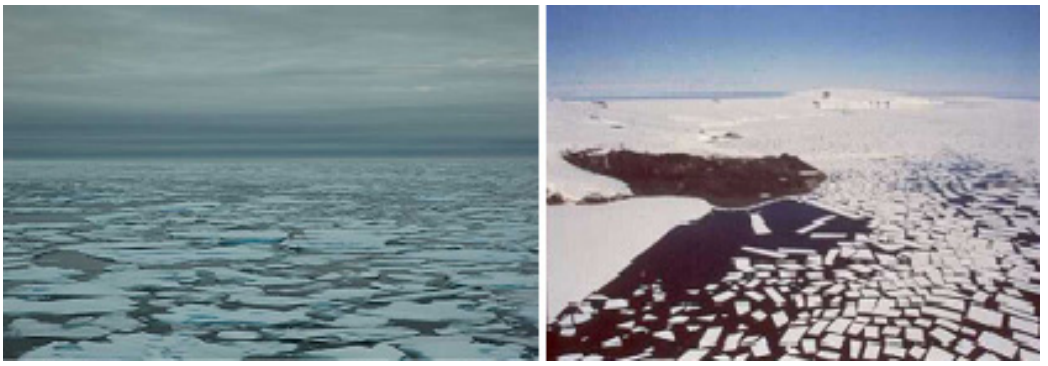

Figure 1: Marginal ice zone in the Antarctic, and wave induced ice fracture at the ice edge. (Courtesy: National Science Foundation, USA, and Squire et al. [5]).

The dominating physical processes that determine ocean surface waves are, input of energy due to wind $S_{i n}$, nonlinear transfer between spectral components due to wave-wave interactions $S_{n l}$, and energy dissipation due to white capping and wave breaking $S_{d s}$. Operational wave models combine these processes in the energy balance equation, which may be written as

$$
\frac{\partial E(f, \theta)}{\partial t}+\mathbf{C}_{\mathbf{g}} \cdot \nabla E(f, \theta)=S_{i n}+S_{d s}+S_{n l}
$$

where the two dimensional wave spectrum $E(f, \theta)$ is a function of frequency $f$, direction $\theta$, time $t$, and position $\mathbf{x}$ and where $\mathbf{C}_{\mathbf{g}}$ is the group velocity. Following Hasselmann et al. [2] and Perrie and $\mathrm{Hu}$ [4], we parameterized $S_{i n}, S_{d s}$, and $S_{n l}$. Figure 2 shows a JONSWAP Wave Energy Spectrum with peak frequency of $0.3 \mathrm{~Hz}$, at $10 \mathrm{~m} / \mathrm{s}$ wind speed developed over 3.25 hours.

We systematically illustrate the mathematical formulations of the ice floe drift velocity due to wind forcing effects. The ice floe trajectories and the ice floe velocity fields are illustrated in a clear cut way. Linearizing the governing equations with zero initial conditions and using the Laplace transform method, we have obtained solutions to simulate the real field conditions. Graphical solutions are displayed in case of the external wind stress which causes the ice floe drift from one place to another. These highly simplified results seem to agree quite well with real field data. Further analysis and detailed investigations of the Eulerian currents, water ice form stress and the wave radiation can be found in [1].

\section{Mathematical formulation}

The mathematical equation of motion for an ice floe in the marginal ice zone due to wind, waves, and current can be written as

$$
m\left(\frac{\partial \mathbf{u}^{\prime}}{\partial t}+\mathbf{f} \times \mathbf{u}^{\prime}\right)=A\left(\boldsymbol{\tau}_{\text {air }}^{\text {skin }}+\boldsymbol{\tau}_{\text {water }}^{\text {skin }}+\boldsymbol{\tau}_{\text {air }}^{\text {form }}+\boldsymbol{\tau}_{\text {water }}^{\text {form }}+\boldsymbol{\tau}_{\text {rad }}^{\text {wave }}\right)-m g \nabla \xi+\mathbf{F}
$$




\section{JONSWAP Spectrum with $f_{\text {peak }}=0.3 \mathrm{~Hz}$}

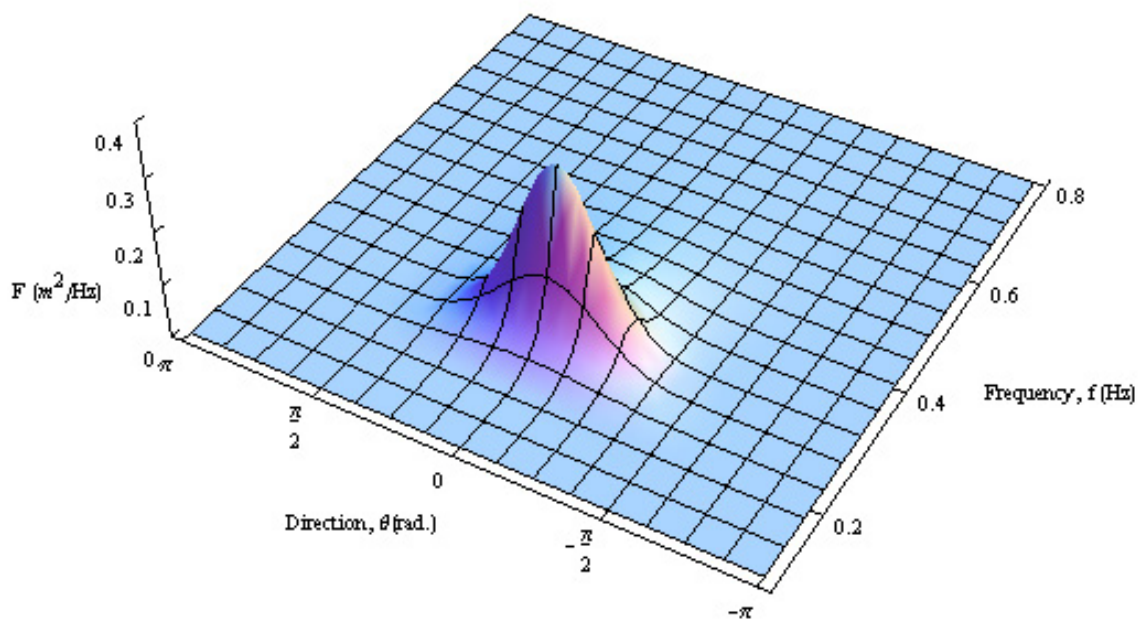

Figure 2: JONSWAP wave energy spectrum.

where $g$ is the acceleration due to gravity, $m$ is the ice mass, $A$ is the ice floe surface area, $\xi$ is the sea surface elevation, $\mathbf{F}$ is the ice internal stress gradient, $\mathbf{u}^{\prime}$ is the absolute ice velocity, $\boldsymbol{\tau}_{\text {air }}^{\text {skin }}$ is the wind stress on the top surface of the ice floe, $\boldsymbol{\tau}_{\text {water }}^{\text {skin }}$ is the water stress on the bottom surface of the ice floe, $\boldsymbol{\tau}_{\text {air }}^{\text {form }}$ is the airice form stress, $\boldsymbol{\tau}_{\text {water }}^{\text {form }}$ is the water-ice form stress, and $\boldsymbol{\tau}_{\text {rad }}^{\text {wave }}$ is the wave radiation pressure.

If the ice concentration is low, the internal stress gradient $\mathbf{F}$ is essentially zero. Replacing $-m g \nabla \xi$ by the geostropic current $m \mathbf{f} \times \mathbf{U}_{\mathbf{g}}$ and neglecting $\mathbf{F}$, equation (2) can be expressed as

$$
m\left(\frac{\partial \mathbf{u}}{\partial t}+\mathbf{f} \times \mathbf{u}\right)=A\left(\boldsymbol{\tau}_{\text {air }}^{\text {skin }}+\boldsymbol{\tau}_{\text {water }}^{\text {skin }}+\boldsymbol{\tau}_{\text {air }}^{\text {form }}+\boldsymbol{\tau}_{\text {water }}^{\text {form }}+\boldsymbol{\tau}_{\text {rad }}^{\text {wave }}\right)
$$

which gives the ice floe velocity, $\mathbf{u}=\mathbf{u}^{\prime}-\mathbf{U}_{\mathbf{g}}$, relative to the geostropic current $\mathbf{U}_{\mathbf{g}}$.

The stresses $\boldsymbol{\tau}_{\text {air }}^{\text {skin }}$ and $\boldsymbol{\tau}_{\text {water }}^{\text {skin }}$ are caused by skin friction. It is assumed that $\left|\boldsymbol{\tau}_{\text {air }}^{\text {form }}\right| \ll\left|\boldsymbol{\tau}_{\text {water }}^{\text {form }}\right|$. Therefore, the final time-dependent equation of motion for an ice floe becomes

$$
\left(\frac{\partial \mathbf{u}}{\partial t}+\mathbf{f} \times \mathbf{u}\right)=\frac{A}{m}\left(\boldsymbol{\tau}_{\text {air }}^{\text {skin }}+\boldsymbol{\tau}_{\text {water }}^{\text {skin }}+\boldsymbol{\tau}_{\text {water }}^{\text {form }}+\boldsymbol{\tau}_{\text {rad }}^{\text {wave }}\right) .
$$

Perrie and $\mathrm{Hu}$ [4] have described these expressions with various types of parameters effecting these stresses. 
In a calm sea condition, the drift of the ice floe can be assumed to be only due to the effect of the wind. The air-ice-skin friction stress $\boldsymbol{\tau}_{\text {air }}^{\text {skin }}$, thus, is usually represented by a quadratic formula in terms of the wind speed $\mathbf{U}_{\mathbf{1 0}}$,

$$
\boldsymbol{\tau}_{\text {air }}^{\text {skin }}=\rho_{a} C_{a i}^{s}\left|\mathbf{U}_{\mathbf{1 0}}-\mathbf{u}\right|\left(\mathbf{U}_{\mathbf{1 0}}-\mathbf{u}\right)
$$

where, $\rho_{a}$ is the air density and $C_{a i}^{s}$ is the air-ice-skin friction drag coefficient. Following Steele et al. [6], we used $C_{a i}^{s} \approx 3 \times 10^{-3}$.

\section{Ice floe drift due to wind stress: model I}

We simplify the governing partial differential equation with the initial condition and obtain,

$$
\begin{aligned}
& \frac{\partial u}{\partial t}-f v=\alpha U_{10}-\alpha u \\
& \frac{\partial v}{\partial t}+f u=-\alpha v
\end{aligned}
$$

where $\mathbf{u}=(u, v, 0)$ are the velocity components of the ice floe in a horizontal plane, $\mathbf{f}=(0,0, f)$ are the Coriolis force components, and $\alpha=\frac{A}{m} \rho_{a} C_{a i}^{s}\left|\mathbf{U}_{\mathbf{1 0}}-\mathbf{u}\right|$. Also, we have $\left(\mathbf{U}_{\mathbf{1 0}}-\mathbf{u}\right)=\left(U_{10}-u,-v, 0\right)$. The wind velocity vector $\mathbf{U}_{\mathbf{1 0}}$ is assumed to be parallel to the positive $x$-direction. Here we assume that $U_{10} \gg$ $|\mathbf{u}|$, i.e., the speed of wind is much greater than that of the ice drift and so we can safely assume that $\alpha$ is a constant parameter. The initial conditions at $t=0$ are assumed as (when there is no wind):

$$
u(0)=0, \quad v(0)=0 .
$$

Using Laplace transform $\mathcal{L}\{u\}=\int_{0}^{\infty} u(t) e^{-s t} d t$ and $\mathcal{L}\{v\}=\int_{0}^{\infty} v(t) e^{-s t} d t$ with the initial conditions (7), the simultaneous differential equations (6) can be transformed as

$$
\begin{aligned}
& (s+\alpha) \mathcal{L}\{u\}-f \mathcal{L}\{v\}=\frac{\alpha U_{10}}{s} \\
& f \mathcal{L}\{u\}+(s+\alpha) \mathcal{L}\{v\}=0
\end{aligned}
$$

Solving these two algebraic equations by Cramer's rule and using residue calculus, we obtain the non-dimensional forms of the solutions as:

$$
\begin{aligned}
& U-a=e^{-\alpha t}\{b \sin f t-a \cos f t\} \\
& V+b=e^{-\alpha t}\{b \cos f t+a \sin f t\}
\end{aligned}
$$

where $U, V, a$, and $b$ are given by

$$
U=\frac{u}{U_{10}}, \quad V=\frac{v}{U_{10}}, \quad a=\frac{\alpha^{2}}{\alpha^{2}+f^{2}} \quad \text { and } \quad b=\frac{\alpha f}{\alpha^{2}+f^{2}} .
$$


Thus, the velocity field of the ice floe, i.e., the $U-V$ plot, can be described by the following circular spiral type solution as a function of time

$$
(U-a)^{2}+(V+b)^{2}=\left(a^{2}+b^{2}\right) e^{-2 \alpha t} .
$$

The equation (10) reveals that the radius of the circle at $t=0$ becomes simply $\sqrt{a^{2}+b^{2}}$ but when the time progresses the radius starts to decrease exponentially and at very large time, i.e., when $t \rightarrow \infty$, the radius of the circle becomes zero implying that the circle shrinks to zero at the center $(a,-b)$. This simulated behavior of the drift of the ice floe is not unusual in a real field situation. The graphical representation of our mathematical model in Fig. 3 confirms this analytical conjecture of the velocity field of the ice floe. The computations were carried out by assuming a cylindrical shape for ice floe with diameter $L$ and a thickness of $T$. The air-ice skin friction drag coefficient was set to $C_{a i}^{s}=3 \times 10^{-3}$, following Steele et al. [6], the Coriolis parameter was set to $f=1.07 \times 10^{-4} \mathrm{~s}^{-1}$, and the wind speeds at $10 \mathrm{~m}$ above the surface $\left(U_{10}\right)$ were varied between $10 \mathrm{~m} / \mathrm{s}$ and $25 \mathrm{~m} / \mathrm{s}$ with $5 \mathrm{~m} / \mathrm{s}$ increments.
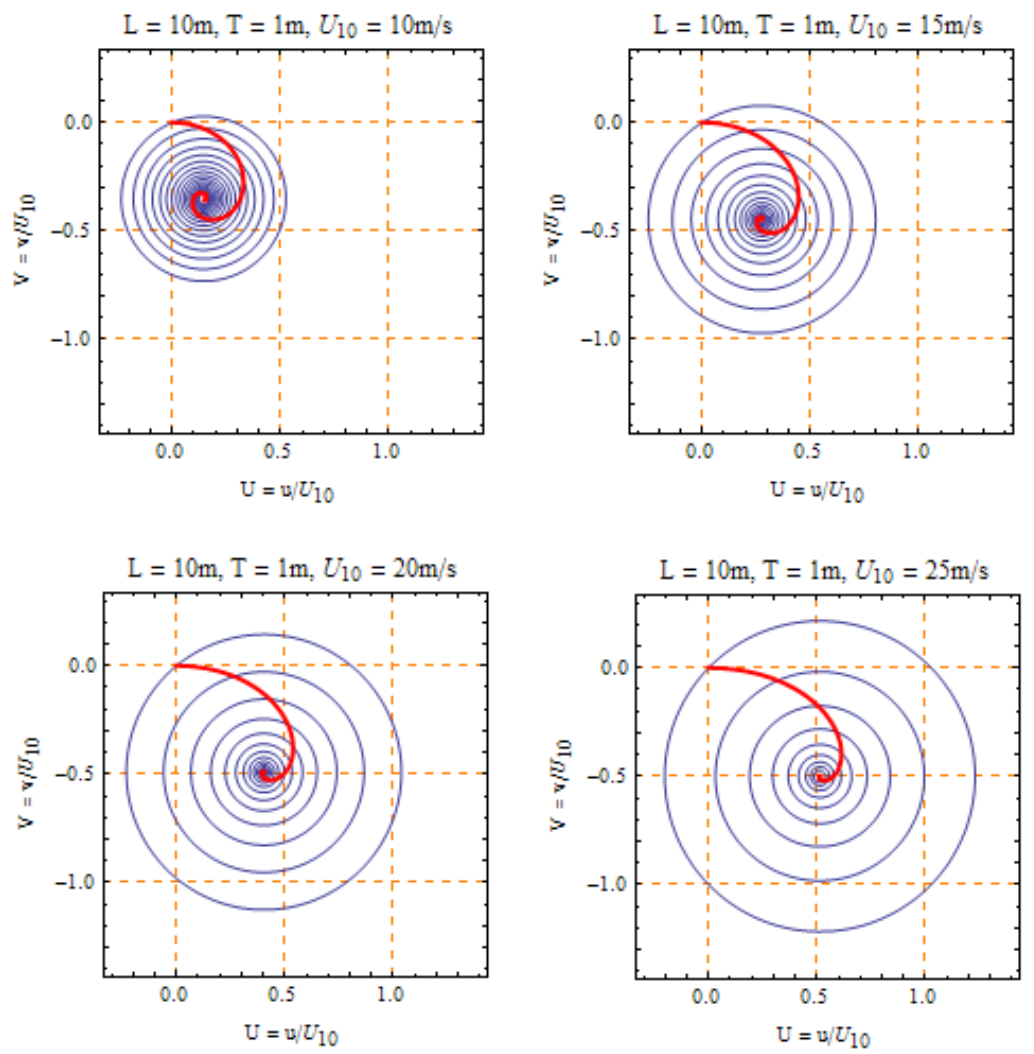

Figure 3: Non-dimensional ice floe velocity field in the phase plane. 
To further verify this conjecture, we determine the ice floe trajectory. We replace $u$ and $v$ in terms of derivatives of $x$ and $y$ with respect to time, such that $u=\frac{\partial x}{\partial t}$ and $v=\frac{\partial y}{\partial t}$. Thus (6) will take the following form:

$$
\begin{aligned}
& \frac{\partial^{2} x}{\partial t^{2}}-f \frac{\partial y}{\partial t}=\alpha U_{10}-\alpha \frac{\partial x}{\partial t} \\
& \frac{\partial^{2} y}{\partial t^{2}}+f \frac{\partial x}{\partial t}=-\alpha \frac{\partial y}{\partial t}
\end{aligned}
$$

The initial conditions are $x(0)=0, \frac{\partial x}{\partial t}(0)=0$. Also, $y(0)=0, \frac{\partial y}{\partial t}(0)=0$. The solutions can be obtained by using the Laplace transform method.

$$
\begin{aligned}
& \left(s^{2}+s \alpha\right) \mathcal{L}\{x\}-f s \mathcal{L}\{y\}=\frac{\alpha U_{10}}{s} \\
& f s \mathcal{L}\{x\}+\left(s^{2}+s \alpha\right) \mathcal{L}\{y\}=0 .
\end{aligned}
$$

Solving these two equations by Cramer's rule and using the residue calculus, we obtain the solutions in non-dimensional forms as

$$
\begin{aligned}
& X(t)=-\frac{\alpha^{2}-f^{2}}{\left(\alpha^{2}+f^{2}\right)}+\alpha t+e^{-\alpha t}\left\{\frac{\left(\alpha^{2}-f^{2}\right) \cos f t-2 \alpha f \sin f t}{\left(\alpha^{2}+f^{2}\right)}\right\} \\
& Y(t)=\frac{2 \alpha f}{\left(\alpha^{2}+f^{2}\right)}-f t-e^{-\alpha t}\left\{\frac{(2 \alpha f) \cos f t+\left(\alpha^{2}-f^{2}\right) \sin f t}{\left(\alpha^{2}+f^{2}\right)}\right\}
\end{aligned}
$$

where $X(t)$ and $Y(t)$ are given by,

$$
X(t)=\frac{x(t)}{\alpha U_{10} /\left(\alpha^{2}+f^{2}\right)} \quad \text { and } \quad Y(t)=\frac{y(t)}{\alpha U_{10} /\left(\alpha^{2}+f^{2}\right)} .
$$

With these definitions, the ice floe trajectories can be obtained as

$$
(X-c)^{2}+(Y-d)^{2}=e^{-2 \alpha t}
$$

where $c=-\frac{\alpha^{2}-f^{2}}{\alpha^{2}+f^{2}}+\alpha t$ and $d=\frac{2 \alpha f}{\alpha^{2}+f^{2}}-f t$, respectively. It can be easily seen that the ice floe path is a circle with the center $(c, d)$ and radius $e^{-\alpha t}$. The parameters $c, d$ and the radius are all dependent on time $t$. Thus the ice floe will move in a circular path with exponentially decreasing radius with respect to time. Further more, for large time, the floe trajectory will follow a linear path with the linearly dependent coordinates of the center of the circle with respect to time. At the initial stage, i.e., at $t=0$, the trajectory will be a unit circle with center at $\left(-\frac{\alpha^{2}-f^{2}}{\alpha^{2}+f^{2}}, \frac{2 \alpha f}{\alpha^{2}+f^{2}}\right)$. The $X-$ coordinate may be positive or negative according to $\alpha^{2}<f^{2}$ or $\alpha^{2}>f^{2}$, respectively. However, the $Y$ - coordinate is always a negative number. The graphical simulations of the non-dimensional trajectory of the ice floe with varying wind speeds and their corresponding phase diagrams following our derived mathematical formulations are shown in Fig. 4. The trajectories 

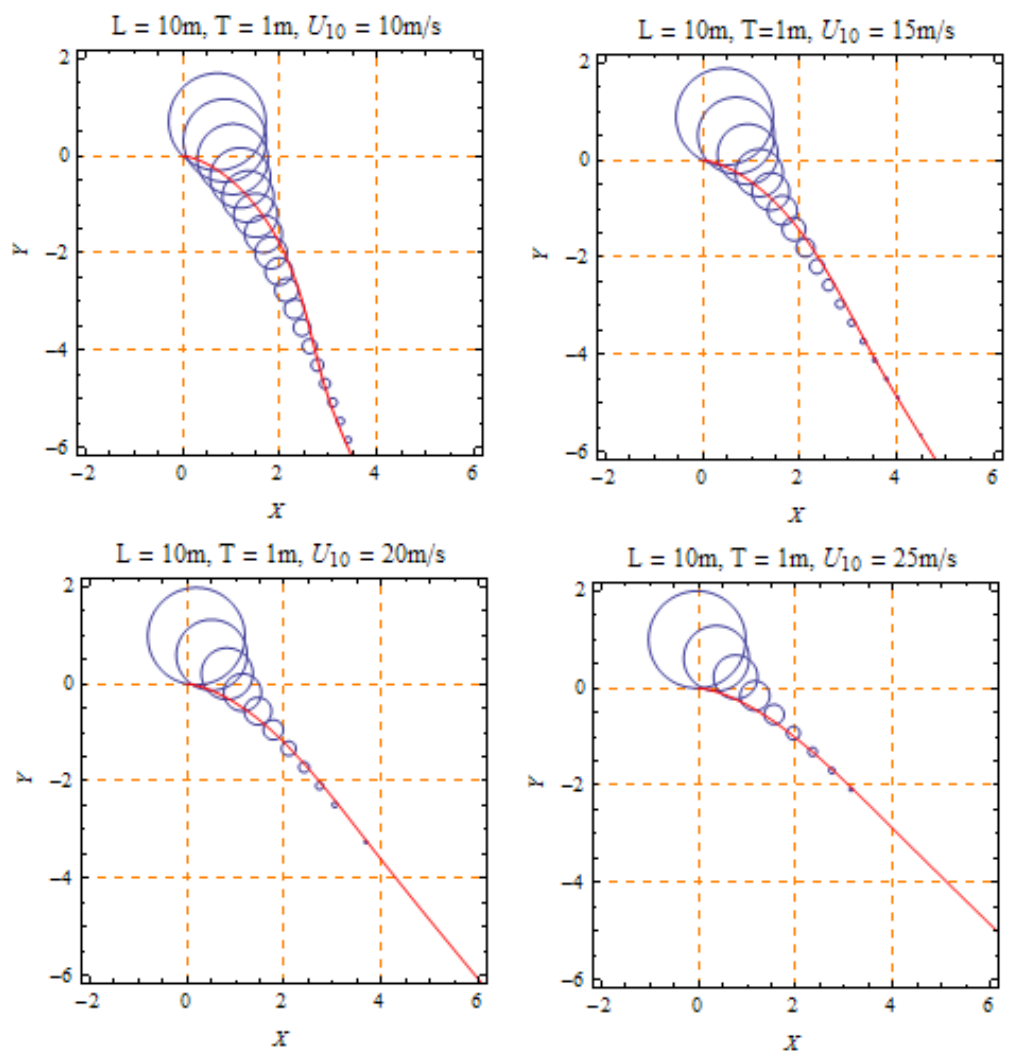

Figure 4: Non-dimensional ice floe trajectories in the phase plane.

are circular spirals starting with a unit circle at $t=0$ and ending with a point circle at $t \rightarrow \infty$; but the center is moving according to law of order $O(\alpha t)$ such that $X \equiv \alpha t$ and $Y \equiv-f t$. Our computation shows that as time passes, the orbital motion of the ice floe, due to the earth's angular motion, gravitational pull, and the constant wind effect, eventually becomes linear. Also, the result of this simplified approach tends to display more displacement along the $y$-axis than that along the $x$-axis. However, this can be corrected with further mathematical computation. One such computation is given below.

\section{Ice floe drift due to wind stress: model II}

The equation of motion of an ice floe (5) can be rewritten in the form,

$$
m\left[\begin{array}{c}
\frac{\partial u}{\partial t} \\
\frac{\partial v}{\partial t} \\
0
\end{array}\right]+\left[\begin{array}{ccc}
i & j & k \\
0 & 0 & f \\
u & v & 0
\end{array}\right]=A \rho_{a} C_{a i}^{s}\left[\begin{array}{c}
\sqrt{\left(U_{10}-u\right)^{2}+v^{2}}\left(U_{10}-u\right) \\
-v \sqrt{\left(U_{10}-u\right)^{2}+v^{2}} \\
0
\end{array}\right]
$$


We obtain,

$$
\left\{\begin{array}{l}
\frac{\partial u}{\partial t}-f v=\frac{A \rho_{a} C_{a i}^{s}}{m}\left(U_{10}-u\right)^{2} \sqrt{1+\frac{v^{2}}{\left(U_{10}-u\right)^{2}}} \\
\frac{\partial v}{\partial t}+f u=-\frac{A \rho_{a} C_{a i}^{s}}{m} v\left(U_{10}-u\right) \sqrt{1+\frac{v^{2}}{\left(U_{10}-u\right)^{2}}}
\end{array}\right.
$$

Using the binomial expansion, the above equations can be written as,

$$
\left\{\begin{aligned}
\frac{\partial u}{\partial t}-f v= & \frac{A \rho_{a} C_{a i}^{s}}{m}\left(U_{10}-u\right)^{2} \\
& \times\left[1+\frac{1}{2}\left(\frac{v}{U_{10}-u}\right)^{2}-\frac{1}{8}\left(\frac{v}{U_{10}-u}\right)^{4}+\frac{1}{16}\left(\frac{v}{U_{10}-u}\right)^{6}+\cdots\right] \\
\frac{\partial v}{\partial t}+f u= & -\frac{A \rho_{a} C_{a i}^{s}}{m} v\left(U_{10}-u\right) \\
& \times\left[1+\frac{1}{2}\left(\frac{v}{U_{10}-u}\right)^{2}-\frac{1}{8}\left(\frac{v}{U_{10}-u}\right)^{4}+\frac{1}{16}\left(\frac{v}{U_{10}-u}\right)^{6}+\cdots\right]
\end{aligned}\right.
$$

Considering wind speed is much greater than the ice floe drift, the above expansion can be linearized in the form,

$$
\left\{\begin{array}{l}
\frac{\partial u}{\partial t}-f v=\frac{A \rho_{a} C_{a i}^{s} U_{10}}{m}\left(U_{10}-2 u\right) \\
\frac{\partial v}{\partial t}+f u=-\frac{A \rho_{a} C_{a i}^{s} U_{10}}{m} v
\end{array}\right.
$$

Proceeding as before, we represent equation (19) in non-dimensional form as follows:

$$
\left\{\begin{array}{l}
\frac{\partial U}{\partial t}-f V=\alpha(1-2 U) \\
\frac{\partial V}{\partial t}+f U=-\alpha V
\end{array}\right.
$$

Using Laplace transform with initial conditions, equation (20) can be transformed as

$$
\left\{\begin{array}{l}
(s+2 \alpha) \mathcal{L}(U)-f \mathcal{L}(V)=\frac{\alpha}{s} \\
f \mathcal{L}(U)+(s+\alpha) \mathcal{L}(V)=0
\end{array}\right.
$$

As before, solving these two equations, we obtain the solutions as,

$$
\left\{\begin{array}{l}
U=\frac{\alpha^{2}}{2 \alpha^{2}+f^{2}}+\frac{e^{-3 \alpha t / 2}}{\beta}\left[-\frac{\beta \alpha^{2}}{2 \alpha^{2}+f^{2}} \cos \left(\frac{\beta}{2} t\right)+\frac{\alpha\left(\alpha^{2}+2 f^{2}\right)}{2 \alpha^{2}+f^{2}} \sin \left(\frac{\beta}{2} t\right)\right] \\
V=-\frac{\alpha f}{2 \alpha^{2}+f^{2}}+\frac{e^{-3 \alpha t / 2}}{\beta}\left[\frac{\alpha \beta f}{2 \alpha^{2}+f^{2}} \cos \left(\frac{\beta}{2} t\right)+\frac{3 \alpha^{2} f}{2 \alpha^{2}+f^{2}} \sin \left(\frac{\beta}{2} t\right)\right]
\end{array}\right.
$$



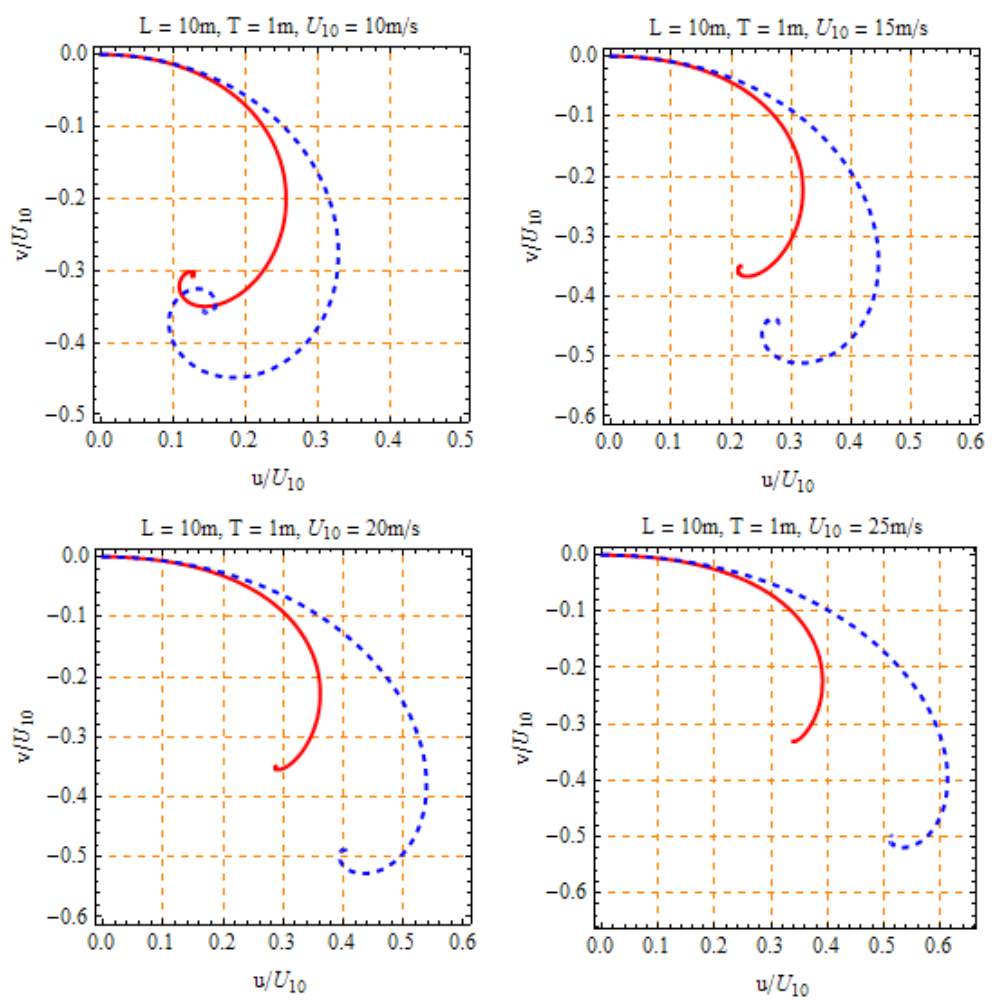

Figure 5: Non-dimensional velocity components of ice floes (dashed line: model I, solid line: model-II).

where $\alpha^{2}-4 f^{2}=-\beta^{2}<0$. The non-dimensional velocity components of the ice floe with the simplifications introduced in Model-I and Model-II have been computed and shown in Fig. 5. Interestingly, Model-II shows further improvements and its computational results tend to agree more closely with those of Perrie and $\mathrm{Hu}$ [4].

\section{Acknowledgement}

We are very grateful to Schneider National, Inc. and Natural Sciences and Engineering Research Council (NSERC) of Canada for financial support leading to this paper.

\section{References}

[1] Abid, R. M., S. H. Mousavizadegan, M. Rahman, Analytical and numerical estimation of ice floe drift in the marginal ice zone, to be published in $J$. of Fluid Mech., 2008. 
[2] Hasselmann, S., K. Hasselmann, G. K. Komen, P. Jenssen, J. A. Ewing, and V. Cardone, The WAM model-A third generation ocean wave prediction model, J. Phys. Oceanogr. 18, 1775-1810, 1988.

[3] Jenkins, A., The use of wave prediction model for driving a near surface current model, Drsch Hydrogr. Z, 42, 134-149, 1989.

[4] Perrie, W and Y. Hu, Air-ice-ocean momentum exchange. Part II: Ice drift, J. Phys. Oceanogr., 27, 1976-1996, 1997.

[5] Squire, Vernon A., John P. Dugan, P. Wadhams, Philip J. Rottier, and Antony J. Liu, Of Ocean Waves and Sea Ice, Annu. Rev. Fluid Mech., 27, 115-168, 1995.

[6] Steele, M., J. H. Morison, and N. Untersteiner, The partition of air-ice-ocean momentum exchange as a function of ice concentration, floe size, and draft . J. Geophys. Res. , 94, 12739-12750, 1994.

[7] Tang, C., and D. Fissel, A simple ice-ocean coupled model for ice drift in marginal ice zones J. Mar. Syst., 2, 465-475, 1991. 